\title{
The Existence and Analysis of Methanol/Methyl Acetate Azeotropes
}

\author{
Alexandre César Balbino Barbosa Filho \\ Department of Chemical Engineering, Federal University of Campina Grande , PB, BRAZIL
}

\begin{abstract}
The objective of the study is to determine and to analyze azeotropic points for the Methanol/ Methyl Acetate Mixture, at different temperatures and to show it colligated variables values on diagram plots. The methodology used was the creation of an algorithm that makes the calculations for a given range of temperature, and the calculations are based on vapor-liquid equilibrium equations. By finding all the azeotropic points, readers will know the limits of temperature where it has or not an azeotrope, with their parameters of pressure and vapor-liquid molar compositions.
\end{abstract}

Keywords: Azeotropes, Computational algorithm, Vapor-liquid equilibrium, Equilibrium thermodynamics

\section{Introduction}

The use of scripting language in the present century is making it easier to obtain results [1], and this is why complex engineering problems are not solved by hand. Azeotropic points are found with the implementation of an algorithm that makes the numerical calculations. This methodology makes this application easier and shareable for the scientific community.

In the azeotrope, the molar composition of the vapor is equal to the molar composition of the liquid, for each chemical species in the mixture [2]. And also, the mixture do not boils at the pure component's boiling point temperature [3]. For the substance " $i$ " [2]:

$$
x_{i}=y_{i}
$$

For binary systems, one correlation for the activity coefficient of two chemical species is given by:

$$
\begin{aligned}
& \ln \left(\gamma_{1}\right)=A \cdot x_{2}^{2} \\
& \ln \left(\gamma_{2}\right)=A \cdot x_{1}^{2}
\end{aligned}
$$

The constant $A$ is a function of the temperature. The relative volatility is a very important parameter to calculate azeotrope points, defined by:

$$
\alpha_{i j}=\frac{y_{i} / x_{i}}{y_{j} / x_{j}}
$$

From modified Raoult's Law:

$$
\frac{y_{i}}{x_{i}}=\frac{\gamma_{i} \cdot P_{i}^{\text {vat }}}{P}
$$

For the binary system and substituting Equation (5) in Equation (4):

$$
\alpha_{12}=\frac{\gamma_{1} \cdot P_{1}^{\text {gat }}}{\gamma_{2} \cdot P_{2}^{\text {vat }}}
$$

For $x_{1}=0$ and $x_{2}=1$, Equations (2) and (3) reduces to:

$$
\begin{gathered}
\gamma_{1}=\exp (A) \\
\gamma_{2}=1
\end{gathered}
$$

For $x_{1}=1$ and $x_{2}=0$, Equations (2) and (3) reduces to:

$$
\begin{aligned}
& \gamma_{1}=1 \\
& \gamma_{2}=\exp (A)
\end{aligned}
$$

Substituting Equations (7) and (8) in Equation (6), and Equations (9) and (10) in Equation (6), are obtained the equations that calculate the upper and lower limits values for the relative volatility, respectively:

$$
\begin{aligned}
& \left(\alpha_{12}\right)_{x_{1}=0}=\frac{P_{1}^{\text {gat }} \cdot \exp (A)}{p_{2}^{\text {gat }}} \\
& \left(\alpha_{12}\right)_{x_{1}=1}=\frac{P_{1}^{\text {gat }}}{p_{2}^{\text {sat }} \cdot \exp (A)}
\end{aligned}
$$

The upper limit value is represented by Equation (11) and the lower limit value is represented by Equation (12), because $\left(\alpha_{12}\right)_{x_{1}=0}>\left(\alpha_{12}\right)_{x_{1}=1}$.

The saturation pressure is represented by Antoine equation trough Equation (13):

$$
P_{i}^{\text {sat }}=\exp \left(a_{i}-\frac{b_{i}}{T+c_{i}}\right)
$$

The value for the relative volatility of an azeotrope is found when the Equation (1) is substituted in Equation (4):

$$
\alpha_{12}=1
$$

The mixture forms an azeotrope in a given temperature if Equation (12) has a value less than a unit and Equation (11) has a value higher than a unit. It can be explained by the fact that $\alpha_{12}$ is a continuous function of $x_{1}$ and has to pass through a unit value on any intermediary composition as it is shown in Equation (14).

Substituting Equation (14) in Equation (6):

$$
\frac{\gamma_{1}^{a z}}{\gamma_{2}^{a z}}=\frac{P_{2}^{\text {sat }}}{P_{1}^{\text {sat }}}
$$

A general relation is established when subtracting the Equation (2) by Equation (3): 


\section{International Journal of Science and Research (IJSR) \\ ISSN (Online): 2319-7064}

Index Copernicus Value (2015): 78.96 | Impact Factor (2015): 6.391

$$
\ln \left(\gamma_{1}\right)-\ln \left(\gamma_{2}\right)=\ln \left(\frac{\gamma_{1}}{\gamma_{2}}\right)=A \cdot\left(1-2 x_{1}\right)
$$

Substituting Equation (15) in Equation (16) and solving for $x_{1}$ :

$$
\begin{gathered}
x_{1}{ }^{a z}=\left(\frac{A-\ln \left(\frac{\gamma_{1}^{a z}}{\gamma_{2}^{a z}}\right)}{2 \cdot A}\right) \\
x_{2}^{a z}=1-x_{1}^{a z}
\end{gathered}
$$

The azeotropic point's pressure equation for the found $x_{1}{ }^{\varpi x}$ with a given temperature, is represented by Equation (19), when substituting Equation (1) and (17) in Equation (5):

$$
P^{a z}=\gamma_{1}^{a z} \cdot P_{1}^{s a t}
$$

All these 19 equations are essential to implement the algorithm and find the points properties, having or not an azeotrope.

\section{Material and Methodology}

The algorithm varies the temperature and calculates the pressure and the molar compositions of possible azeotropic points, existing on the given temperature. For doing the calculations, there are necessary some parameters of the saturation pressure for the Methanol (1) and Methyl Acetate (2) [4],[5], as follows Table 1:

Table 1: Parameters for the saturation pressure of Methanol and Methyl Acetate

\begin{tabular}{|c|c|}
\hline \multicolumn{2}{|c|}{ Saturation pressure's parameters } \\
\hline $\mathrm{A}_{1}$ & 16.59158 \\
\hline $\mathrm{B}_{1}$ & 3643.31 \\
\hline $\mathrm{C}_{1}$ & 33.424 \\
\hline $\mathrm{A}_{2}$ & 14.25326 \\
\hline $\mathrm{B}_{2}$ & 2665.54 \\
\hline $\mathrm{C}_{2}$ & 53.424 \\
\hline
\end{tabular}

It was used the software MATLAB ${ }^{\circ}(\mathrm{R} 2013 \mathrm{a}$, Mathworks, Natick, MA, USA) for making the implementation of the algorithm. The calculations were made for each temperature with a loop in the algorithm, which was varied the temperature's value, starting with a value of $109 \mathrm{~K}$ until 500 $\mathrm{K}$ with a step size of 0.1 .

\subsection{Algorithm's Methodology}

The methodology of the algorithm used for this binary mixture is:

Step 1: Calculate the saturation pressure for the substances, and the constant $A$ [5] whose appears in Equations (2) and (3), for the given temperature. For the Methanol/Methyl Acetate mixture:

$$
A=2.771-0.00523 \cdot T
$$

Step 2: Calculate the upper and lower limits values for the relative volatility with Equations (11) and (12);
Step 3: If the lower limit value calculated in Step 2 is less or equal to 1 and the upper limit value is greater or equal to 1 , then:

1) Calculate the ratio $\frac{r_{1}^{a x}}{f_{2}^{a x}}$ through Equation (15) by substituting the saturation pressures' values calculated in Step 1;

2) Calculate the natural logarithm of this ratio;

3) Calculate through Equations (17), (18) and (1), the molar compositions of the Methanol/Methyl Acetate mixture in the azeotrope;

4) Calculate methanol's activity coefficient through Equation (2);

5) Calculate the azeotrope's pressure through Equation (19);

6) Store the calculated values of the azeotrope's pressure and molar compositions.

Step 4: If Step 3 is not true, go back to Step 1 with the next temperature value;

Step 5: After Step 3 is executed, go back to Step 1 with the next temperature value.

Repeat these steps until the reach of the last chosen value for the temperature, which is in this particular case, $600 \mathrm{~K}$. And then, plot $x_{1}{ }^{a z}$ vs $\mathrm{T}, x_{2}{ }^{a z}$ vs $\mathrm{T}$ and $P^{a z}$ vs $\mathrm{T}$, with the stored values from Step 3.

\section{Results and Discussions}

\subsection{Analysis of the azeotropic pressure and temperature}

The binary mixture of Methanol and Methyl Acetate have azeotropes only in a range of 109 to $461.2 \mathrm{~K}$. Figures 1,2 and 3 relates the azeotropic point's properties (pressure and molar compositions) for different temperatures in the vaporliquid equilibrium (VLE). The behavior of the pressure in the existing azeotropes, varying with respect of temperature, is shown below in Figure 1:

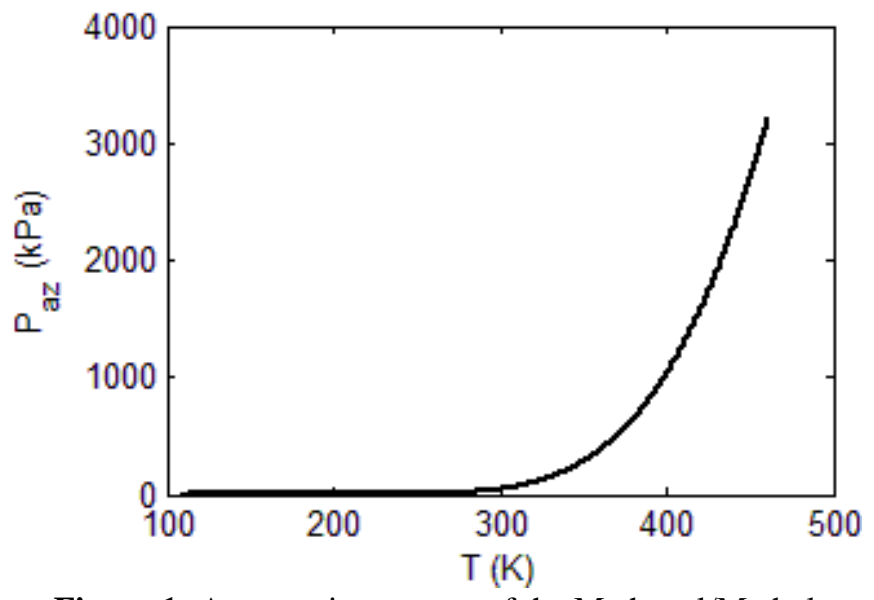

Figure 1: Azeotropic pressure of the Methanol/Methyl Acetate mixture in the azeotropes.

As it can be seen in Figure 1, the pressure in the azeotropic points, have a non-linear relation with the temperature. It can be explained by the activity coefficient model for this binary mixture, and with the use of Equation (19). The maximum azeotropic pressure is $3213.32 \mathrm{kPa}$, whereas the minimum is 


\section{International Journal of Science and Research (IJSR) \\ ISSN (Online): 2319-7064}

Index Copernicus Value (2015): 78.96 Impact Factor (2015): 6.391

almost equal to $0 \mathrm{kPa}$. The pressure in the azeotropic points has an exponential behavior due to temperature, establishing consent with Equation (19).

If the binary mixture of methanol and methyl acetate, on its state changing of pressure and temperature during an operation, reach one of these points shown in Figure 1, it is formed an azeotrope. At this moment, the mixture begins to behave as if it were a pure substance, hindering the separation by simple distillation.

Once it is formed an azeotrope, the corresponding molar compositions of each mixture components, for an azeotropic point shown in Figures 2 and 3, are linked to its azeotropic pressure and temperature shown in Figure 1.

\subsection{Analysis of the azeotropic molar compositions}

The molar compositions in the existing azeotropes for the Methanol/Methyl Acetate mixture, varying with respect of temperature, are respectively shown in Figures 2 and 3:

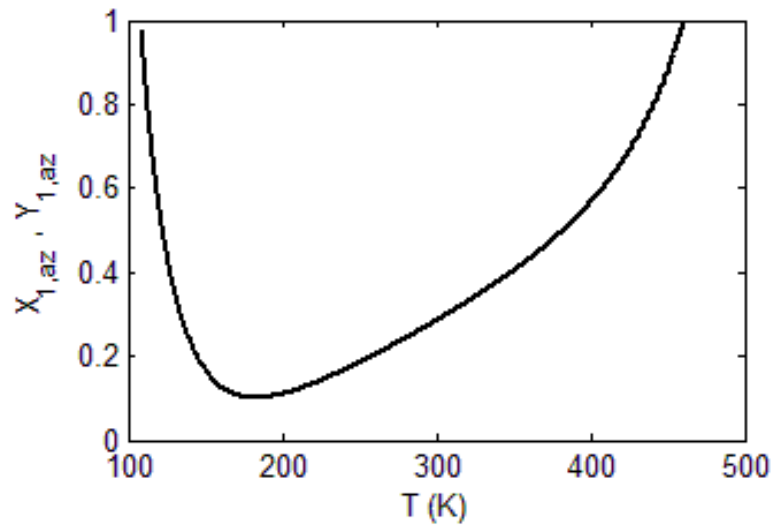

Figure 2: Molar compositions of the methanol in the azeotropes.

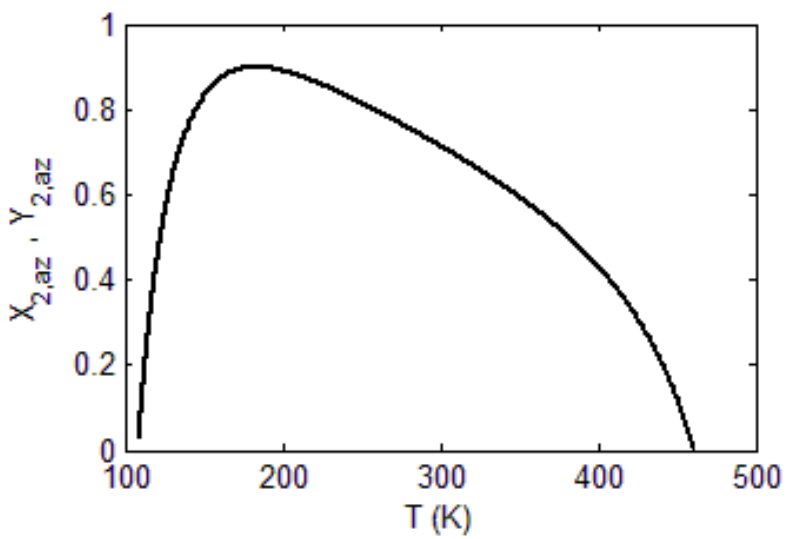

Figure 3: Molar compositions of the methyl acetate in the azeotropes.

The composition of the Methanol in the azeotrope shown in Figure 2, decreases in the direction of temperature change from 109 to $183.8 \mathrm{~K}$, while it increases in the direction of temperature change from 183.8 to $461.2 \mathrm{~K}$. As the rest of the composition belongs to Methyl Acetate as shown in Figure 3, its behavior is the inverse of the one represented by Methanol in this mixture. Such behaviors are justified by the mixture's empiric model.

\section{Conclusion}

For the Methanol/Methyl Acetate mixture, the molar compositions have an inflection point at $183.8 \mathrm{~K}$, and the existing limits for the azeotropic pressure and temperature are respectively, $0-3213.32 \mathrm{kPa}$ and $109-461.2 \mathrm{~K}$. The algorithm's methodology can be used for others binary mixtures.

\section{Future Scope}

Do the algorithm's methodology of this present paper for many others binary mixtures, generating a table with the upper and lower limits of the azeotropic pressures and temperatures. If these binary mixtures do not have azeotropes, it must be also reported.

\section{References}

[1] Ousterhout, J. K. (1998). Scripting: Higher level programming for the 21st century. Computer, 31(3), 2330. Available from: http://ieeexplore.ieee.org/document/660187 [Accessed 14th August 2017]

[2] Smith, J. M., Van Ness, H. C., Abbott, M. M., \& Queiroz, E. M. (2000). Introdução à termodinâmica da engenharia química. LTC.

[3] Hilmen, E. K. (2000). Separation of azeotropic mixtures: tools for analysis and studies on batch distillation operation. Available from: http://folk.ntnu.no/skoge/publications/thesis/2000_hilmen /Thesis_Hilmen.pdf [Accessed 16th august 2017]

[4] Poling, B. E., Prausnitz, J. M., \& O'connell, J. P. (2001). The properties of gases and liquids (Vol. 5). New York: Mcgraw-hill.

[5] Gmehling, J., Onken, U., Arlt, W., Grenzheuser, P., Weidlich, U., Kolbe, B., \& Rarey, J. (1986). Chemistry Data Series, Volume I Vapor-Liquid Equilibrium Data Collection. DECHEMA, Frankfurt, Germany. Available from: http://dechema.de/en/Volume+I.html [Accessed 19th September 2017]

\section{Author Profile}

Alexandre César Balbino Barbosa Filho is in his last year of chemical engineering graduation at Federal University of Campina Grande, Campina Grande, PB, Brazil. Alexandre have been actuating in the areas of modeling, simulation, optimization and control of processes, and development of processes design. Currently doing an internship in the sugar fabrication's sector in Usina Santo Antonio, São Luiz do Quitunde -AL. 